This document is the accepted manuscript version of the following article: Meng, K. Y., Ahmed, A. S., Baćani, M., Mandru, A. O., Zhao, X., Bagués, N., Esser, B. D., Flores, J., McComb, D. W., Hug, H. J., \& Yang, F. (2019). Observation of nanoscale skyrmions in SrIro3/SrRuO3 bilayers. Nano Letters, 19(5), 3169-3175. https://doi.org/10.1021/acs.nanolett.9b00596

\title{
Observation of Nanoscale Skyrmions in
}

\section{$\mathrm{SrIrO}_{3} / \mathrm{SrRuO}_{3}$ Bilayers}

Keng-Yuan Meng ${ }^{1,+}$, Adam S. Ahmed ${ }^{1,+}$, Mirko Baćani ${ }^{2}$,Andrana-Oana Mandrü, Xue Zhao ${ }^{2}$, Nuria Bagués Salguero ${ }^{3}$, Bryan D. Esser ${ }^{3}$, Jose Flores ${ }^{1}$, David W. McComb ${ }^{3}$, Hans J. Hug ${ }^{2,4}$ and Fengyuan Yang ${ }^{1, *}$

${ }^{1}$ Department of Physics, The Ohio State University, Columbus, OH, 43210, USA

${ }^{2}$ Empa, Swiss Federal Laboratories for Materials Science and Technology, CH-8600 Dübendorf, Switzerland

${ }^{3}$ Center for Electron Microscopy and Analysis, The Ohio State University, Columbus, OH, 43210, USA

${ }^{4}$ Department of Physics, University of Basel, CH-4056 Basel, Switzerland

KEYWORDS: skyrmions, topological Hall effect, magnetic force microscopy, epitaxial oxide films.

ABSTRACT. Skyrmion imaging and electrical detection via topological Hall (TH) effect are two primary techniques for probing magnetic skyrmions which hold promise for next-generation magnetic storage. However, these two kinds of complementary techniques have rarely been employed to investigate the same samples. We report the observation of nanoscale skyrmions in $\mathrm{SrIrO}_{3} / \mathrm{SrRuO}_{3}(\mathrm{SIO} / \mathrm{SRO})$ bilayers in a wide temperature range from 10 to $100 \mathrm{~K}$. The $\mathrm{SIO} / \mathrm{SRO}$ 
bilayers exhibit remarkable TH effect which is up to $200 \%$ larger than the anomalous Hall (AH) effect at $5 \mathrm{~K}$, and zero-field $\mathrm{TH}$ effect at $90 \mathrm{~K}$. Using variable-temperature, high-field magnetic force microscopy (MFM), we image skyrmions as small as $10 \mathrm{~nm}$, which emerge in the same field ranges as the $\mathrm{TH}$ effect. These results reveal a rich space for skyrmion exploration and tunability in oxide heterostructures.

\section{Introduction}

The fast-growing information technology demands a paradigm shift in data storage. Skyrmions are promising in that regard as they can be as small as $1 \mathrm{~nm}^{1}$, exist at room temperature ${ }^{2-}$ ${ }^{5}$, require much smaller energy to manipulate than current technology ${ }^{6}$, and are robust to perturbations owing to their topological protection. However, in order to make skyrmions a viable next-generation technology, all these properties need to be unified in one material system, which remains elusive. There are two determining factors for skyrmion size: the exchange energy $(J)$ and the Dzyaloshinskii-Moriya interaction (DMI) (D). Bulk crystals, such as FeGe, host skyrmions stabilized by broken bulk inversion symmetry ${ }^{7}$. Mirror symmetry breaking can also give rise to DMI, which has been explored in metallic multilayer systems ${ }^{8}$ to tune both $J($ e.g., Co) and interfacial $D($ e.g., Pt). The metallic multilayers have yielded skyrmions from $\sim 1 \mu \mathrm{m}$ down to $27 \mathrm{~nm}^{9,10}$.

Epitaxial oxide heterostructures offer a fertile playground to explore novel skyrmion materials because of their high crystalline quality and tunable electronic and magnetic properties via epitaxial strain, doping, thickness, oxygen vacancies, and octahedral rotations ${ }^{11-15}$. Moreover, oxides are the only class of materials to have stabilized skyrmions in both conducting ${ }^{16}$ and insulating ${ }^{17}$ electronic phases.

\section{Experimental Details}


Bilayer (BL) epitaxial films of 2 unit cell (uc) thick $\mathrm{SrIrO}_{3}$ on $\mathrm{SrRuO}_{3}(10 \mathrm{uc}$ ) (labeled as "10 uc BL", $1 \mathrm{uc} \approx 0.39 \mathrm{~nm}$ ) were grown on (001)-oriented $\mathrm{SrTiO}_{3}$ (STO) substrates using offaxis sputtering ${ }^{18-20}$. The excellent film quality is confirmed by X-ray diffraction and scanning transmission electron microscopy results (see Supplementary Information).

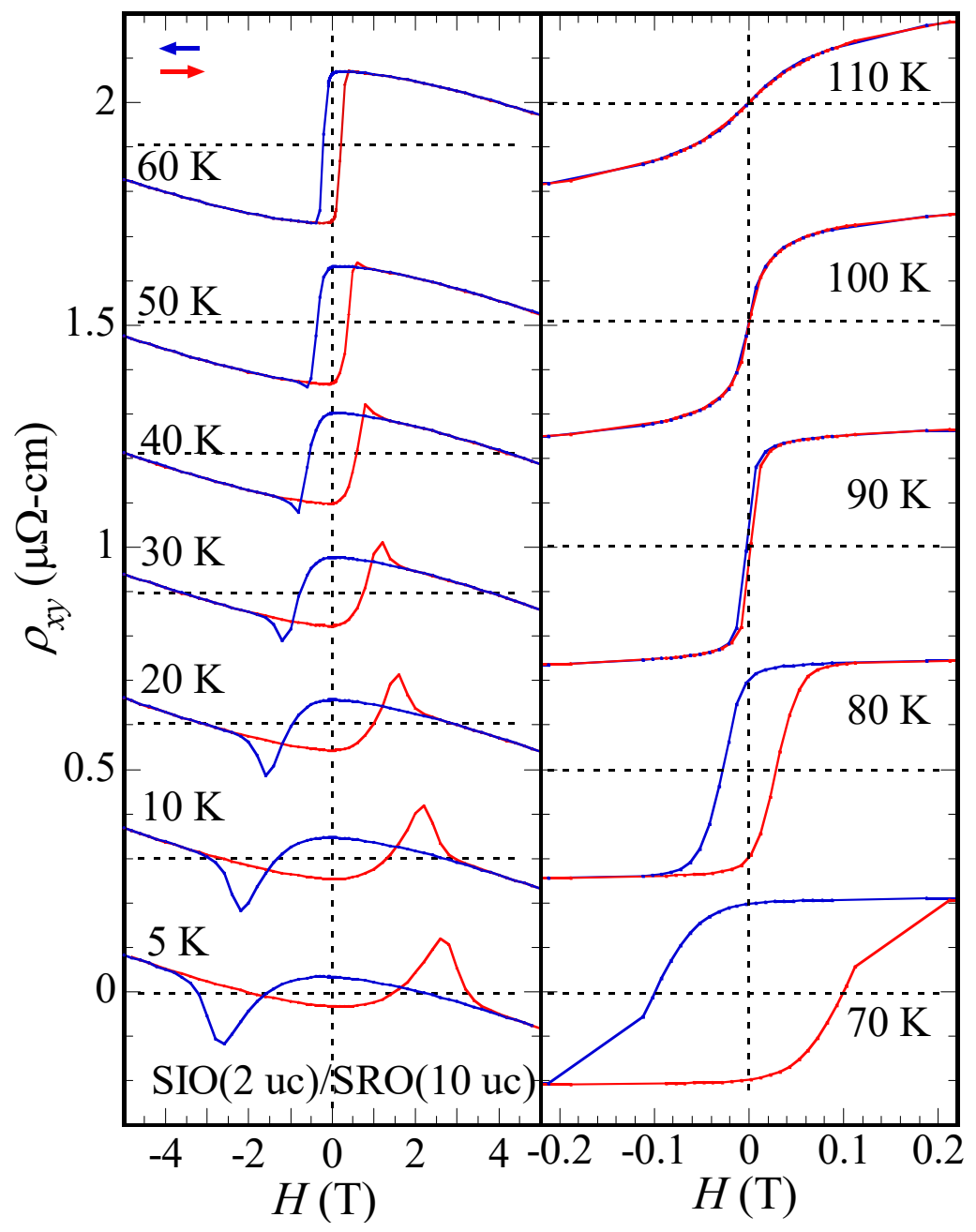

Figure 1. Hall resistivity of a SIO(2 uc)/SRO(10 uc) bilayer on STO(001). Pronounced topological Hall peaks (e.g., at $H= \pm 2.6 \mathrm{~T}$ at $5 \mathrm{~K}$ ) are present from 5 to $50 \mathrm{~K}$.

The existence of skyrmions in SIO/SRO bilayers was first proposed by Matsuno et al. ${ }^{11}$ based on a TH effect in the bilayers. We first investigate our 10 uc BL sample by electrical Hall measurement as shown in the Hall resistivity $\left(\rho_{x y}\right)$ hysteresis loops in Fig. 1 at temperatures $(T)$ from 5 to $110 \mathrm{~K}$. At $T=5 \mathrm{~K}$, pronounced peaks occur near the switching of $\rho_{x y}$. As the 
temperature increases. the magnitude of the peaks near the coercive field $\left(H_{\mathrm{c}}\right)$ of the Hall loop decreases and disappears at $T>50 \mathrm{~K}$.
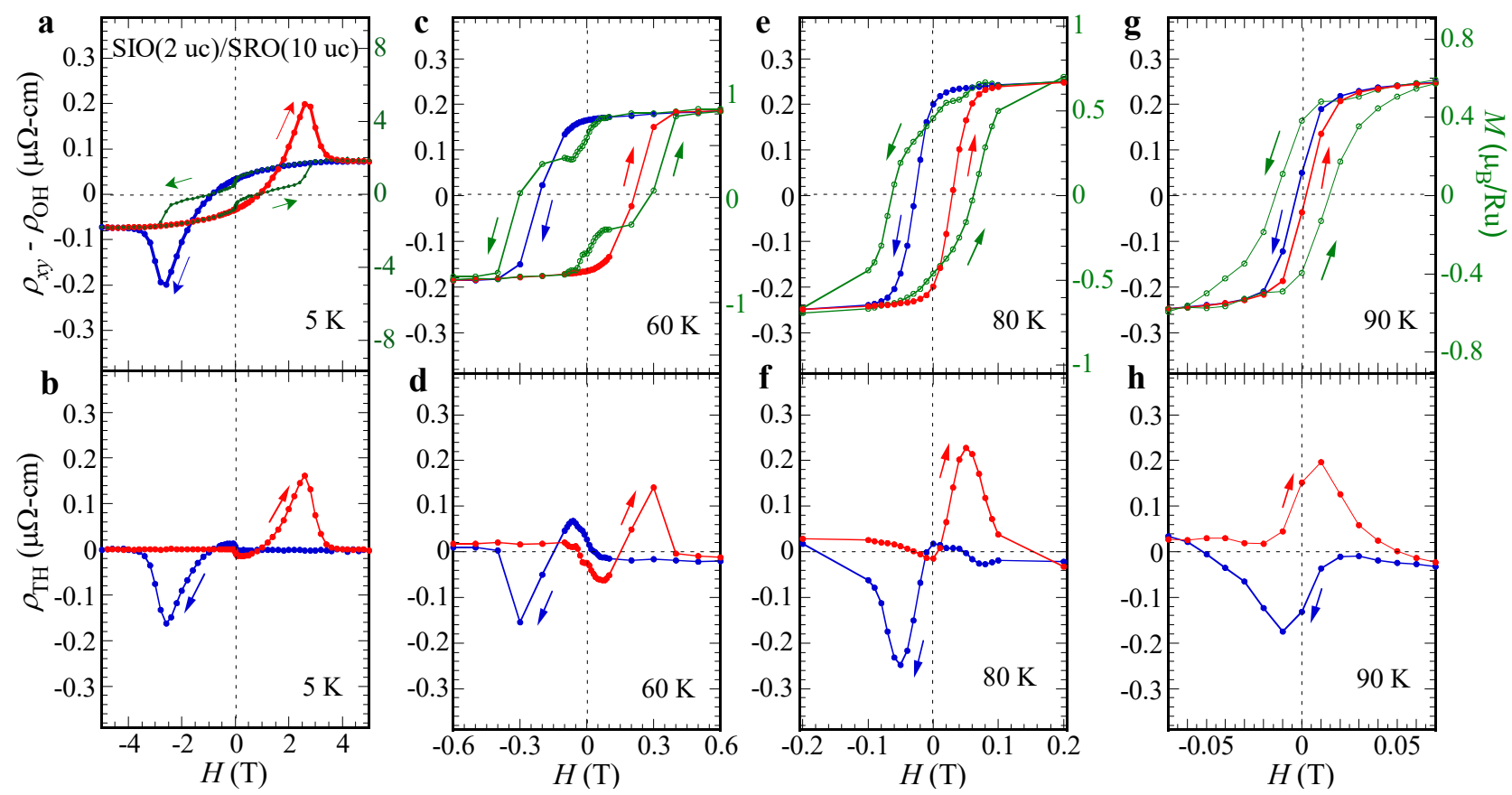

Figure 2. Extraction of topological Hall resistivity of a $\operatorname{SIO}(2 \mathrm{uc}) / \mathrm{SRO}(10 \mathrm{uc})$ bilayer on STO(001). Hall resistivity after subtracting the ordinary Hall contribution, and magnetization hysteresis loops at a, $5 \mathrm{~K}, \mathbf{c}, 60 \mathrm{~K}, \mathbf{e}, 80 \mathrm{~K}$, and $\mathbf{g}, 90 \mathrm{~K}$, all with out-of-plane fields. Clear differences between the coercive fields of $\rho_{x y}(H)$ and $M(H)$ at 60,80 , and $90 \mathrm{~K}$ is observed. Extracted topological Hall resistivity $\left(\rho_{\mathrm{TH}}\right)$ at $\mathbf{b}, 5 \mathrm{~K}, \mathbf{d}, 60 \mathrm{~K}, \mathbf{f}, 80 \mathrm{~K}$, and $\mathbf{h}, 90 \mathrm{~K}$. Zero-field $\rho_{\mathrm{TH}}$ is clearly demonstrated at $90 \mathrm{~K}$.

There are three contributions to the Hall data: the ordinary Hall $(\rho \mathrm{OH})$, anomalous Hall $\left(\rho_{\mathrm{AH}}\right)$, and topological Hall $\left(\rho_{\mathrm{TH}}\right)$ resistivities given by ${ }^{21}, \rho_{x y}=\rho_{\mathrm{OH}}+\rho_{\mathrm{AH}}+\rho_{\mathrm{TH}}$, where $\rho_{\mathrm{OH}}$ is proportional to field, $\rho_{\mathrm{AH}} \propto M$ and $M$ is the out-of-plane magnetization. To extract the TH contribution, we use magnetization hysteresis loops as references to subtract out the anomalous Hall resistivity. Figures $2 \mathrm{a}, 2 \mathrm{c}, 2 \mathrm{e}$, and $2 \mathrm{~g}$ show the field dependencies of $\rho_{x y}-\rho$ OH together with the magnetization hysteresis loops (scaled to match the Hall loops at saturation) for the $10 \mathrm{uc} \mathrm{BL}$ at 5, 60, 80, and $90 \mathrm{~K}$, respectively (see also the Supplementary Information). Since our magnetoresistance is small $(<2 \%)$ at all temperatures, the magnetization hysteresis loops represent 
the AH contribution to the total Hall resistivity ${ }^{22}$. In Fig. 2a, the Hall loop at $5 \mathrm{~K}$ shows pronounced peaks at $H_{\mathrm{c}}$ of the magnetization loop, where the maxima in $\rho_{x y}$ are $\sim 200 \%$ larger than $\rho_{\mathrm{AH}}$ at high fields. These $\rho_{x y}$ peaks can be attributed to the TH effect due to the presence of skyrmions (see MFM images below). After subtracting the AH contribution, we obtain the TH resistivity as shown in Fig. 2b.

Although the TH peaks disappear at $T>50 \mathrm{~K}$, the Hall loops and magnetizations loops are clearly separated with very different values of $H_{\mathrm{c}}$ as shown in Fig. 2. The difference in coercivity between the Hall and magnetization hysteresis loops indicates that $\mathrm{TH}$ resistivity is still present without the peak feature at $T>50 \mathrm{~K}$. The $\mathrm{TH}$ resistivity after subtracting the $\mathrm{AH}$ contribution persists from 5 to $90 \mathrm{~K}$ as shown in Figs. $2 \mathrm{~b}, 2 \mathrm{~d}, 2 \mathrm{f}$, and $2 \mathrm{~h}$. We note that the step-like features near zero-field in the magnetization loops in Figs. $2 \mathrm{a}$ and $2 \mathrm{c}$ are artifacts that do not appear in the Hall data; thus, the features near zero field in Figs. $2 b$ and $2 d$ are not due to the skyrmion-induced topological Hall effect (see Supplementary Information for further discussion). At $90 \mathrm{~K}, \rho_{\mathrm{TH}}$ is peaked at \pm 100 Oe with a maximum of $0.2 \mu \Omega \cdot \mathrm{cm}$ and has a large value of $0.15 \mu \Omega \cdot \mathrm{cm}$ at zero field, which is much larger than those in metallic multilayers ${ }^{23}$, and comparable to those of FeGe films ${ }^{22,24}$. This is the first observation of zero-field TH effect in the oxide films.

We attribute the pronounced TH effect to the existence of skyrmions. The skyrmion size can be estimated from the areal density ${ }^{25,26}, \rho_{\mathrm{TH}, \max }=P R_{o} \phi_{o} n_{s k}$, where $\rho_{\mathrm{TH}, \max }$ is the maximum in $\rho_{\mathrm{TH}}, P$ is the spin polarization in $\mathrm{SrRuO}_{3}(-9.5 \%)^{11,27}, \phi_{o}$ is the flux quantum, $R_{o}$ is the ordinary Hall coefficient, and $n_{s k}$ is the areal density of skyrmions. Though this equation is strictly true for skyrmion lattices, we use it as a rough estimate to obtain a skyrmion length scale. From the $5 \mathrm{~K}$ data, the skyrmion size is calculated to be $6.3 \mathrm{~nm}$ in diameter. For the $90 \mathrm{~K}$ data, the estimated skyrmion diameter is about $2.4 \mathrm{~nm}$. We note that Kan et al. ${ }^{15}$ observed peaks in the Hall 
measurement in their $\mathrm{SrRuO}_{3} / \mathrm{NdGaO}_{3}$ samples, which was attributed to inhomogeneous magnetoelectric properties, instead of TH effect. However, as shown below, we will correlate the pronounced Hall peaks in our SIO/SRO bilayers with the skyrmions imaged in the MFM data in the same field-temperature region, confirming that those peaks are from topological Hall effect.

In order to independently confirm the existence of skyrmions in our SIO/SRO bilayer, high-resolution MFM imaging was performed at various temperatures and over a field range spanning the relevant regions of the TH data. Figure 3 shows background-subtracted MFM images and cross-sections acquired at $10 \mathrm{~K}$ at different magnetic fields. Additional details on the origin of the background and the subtraction process are given in the Supplementary Information.

The sample was zero-field cooled from room temperature to $10 \mathrm{~K}$. The MFM image taken at zero field (Fig. 3a) shows a maze pattern of up (red) and down (blue) domains corresponding to a negative and positive shift, respectively, of the cantilever resonance frequency (f), for an initial magnetization of the MFM tip in the up direction. Note that Figs. 3a-3d are coded with a (reversed) color scale showing red color for up domains leading to a negative $\Delta \mathrm{f}$ contrast (attractive magnetic force, Fig. 3e). In Fig. 3a, the zero-field cooled magnetic state is shown and exhibits a labyrinth domain structure. When a positive (up) field of $2250 \mathrm{mT}$ is applied (Fig. 3b), the red (up) domains expand at the expense of the blue (down) domains. When the domain size is larger than the film thickness, the field in the center of a domain decays as the domain size increases ${ }^{28}$, and the MFM $\Delta \mathrm{f}$-contrast in the center of the domain becomes smaller (less red) as the domain expands. For this reason only the shrinking blue (down) domains are visible as the red domains expand in Figs. 3c and 3d. MFM images at higher fields (not shown here) show a further expansion/shrinking of the $\mathrm{red} / \mathrm{blue}$ domains, with saturation obtained at $3100 \mathrm{mT}$.

After saturation in positive fields, further MFM data were acquired at negative fields 
through a magnetic reversal process (Figs. 3g-31), where a more conventional color scale has been chosen, i.e., red denotes a positive $\Delta \mathrm{f}$ contrast (repulsive magnetic force, see Fig. 3f). The domain reversal becomes apparent when comparing the blue domain at $2450 \mathrm{mT}$, highlighted by the ellipsis in Fig. 3c, to the re-appearing of the same domain in red at $-2450 \mathrm{mT}$ in Fig. 3j.

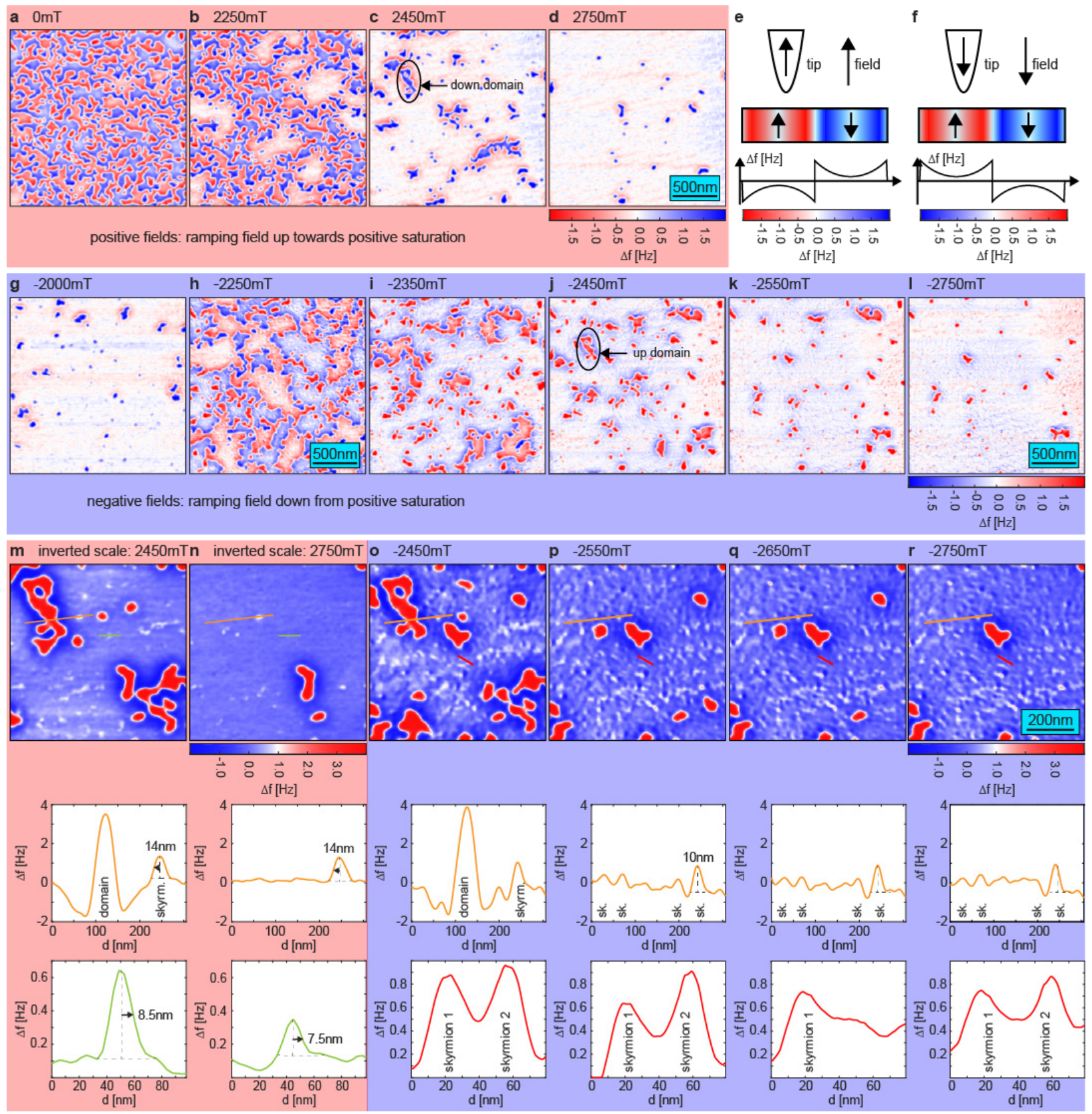

Fig. 3 | Background-subtracted MFM data recorded at $10 \mathrm{~K}$ at different fields for the $\mathrm{SIO}(2$ uc)/SRO(10 uc) bilayer. a-d, Evolution of the as-grown domain structure at 0, 2250, 2450, and $2750 \mathrm{mT}$. Schematics illustrating that in e, red/blue domains have an up/down magnetization and 
generate a negative/positive frequency shift because the initial tip magnetization is up; and $\mathbf{f}$, after application of negative fields the tip magnetization is down. $\mathbf{g}-\mathbf{l}$, MFM images acquired at negative fields of -2000 to $-2750 \mathrm{mT}$. Note that because the tip magnetization is flipped to the down direction, red/blue colors now denote domains with a down/up magnetization direction. This change of contrast becomes apparent at the location of the domain highlighted by the ellipse in panel (c) taken at $+2250 \mathrm{mT}$ and $(\mathbf{j})$ taken at $-2250 \mathrm{mT}$ that appears with almost the same shape on the up and down branches of the hysteresis loop. $\mathbf{m}, \mathbf{n}$, High-magnification views of the MFM images taken at positive fields [inverted scales of (c) and (d)], which are compared to the corresponding images taken at negative fields $[(\mathbf{o})$ and $(\mathbf{q})]$. p, r, Additional negative field data taken at -2550 and $-2750 \mathrm{mT}$. The bottom rows of panels $(\mathbf{m})$ through (r) display cross-sections taken at the location marked by the orange, green, and red lines plotted into the images above.

For $|\mathrm{B}|<2250 \mathrm{mT}$ (not shown) only very minor changes of domain structures occur, i.e., the contrast is similar to the saturated magnetic state with $\Delta \mathrm{f}$ near zero. For $|\mathrm{B}|>2250 \mathrm{mT}$ the maze domain pattern changes significantly and smaller bubble-shaped domains appear (note we do not use the term skyrmion here, because we reserve this term for bubble domains with a small radius which would not exist in the absence of DMI). The field range from 2250 to $2450 \mathrm{mT}$ coincides with that where the largest $\mathrm{TH}$ effect occurs. At higher temperatures, similar bubble domains are observed at significantly smaller fields (see MFM data for higher temperatures at 30, 60 , and $100 \mathrm{~K}$ in Supplementary Information), again matching the fields of the largest TH effect at the corresponding temperatures.

It is noteworthy that the TH signal appears only on the nucleation branch of the hysteresis curve, where the magnetic reversal occurs. Figures $3 \mathrm{~m}$ and $3 \mathrm{n}$ compare high-magnification views of the MFM data taken at positive fields to the corresponding images taken at negative fields (Figs. 3o and 3r). To facilitate the comparison of MFM data taken at opposite fields, the color coding of the MFM data acquired at positive fields (Figs. 3m and 3n) have been inverted. Three observations need to be discussed.

First, at negative fields (Fig. 3o), several domains re-appear with almost identical shape at the locations where they were observed in positive fields (Fig. 3m), although the sample was saturated at $7 \mathrm{~T}$ (no domains are visible above $3100 \mathrm{mT}$, not shown). Such a high degree of return 
or complementary point memory indicates that the domain formation in the thin bilayer must be strongly determined by the pinning sites, or locally enhanced values of DMI. ${ }^{29}$

Second, corresponding to the occurrence of the TH signal on the nucleation branch of the magnetization loop, a much larger number of very small bubble domains with a radius below 20 $\mathrm{nm}$ are visible at negative fields (Figs. 3o-3r) than at positive fields (Figs. 3m and 3n). Because of their small size, these bubble domains generate a much weaker field at a tip height of $10.5 \mathrm{~nm}$ above the sample surface and consequently, a small $\Delta \mathrm{f}$ (fractions of a $\mathrm{Hz}$ ) as compared to that for larger domains, which can be up to $4 \mathrm{~Hz}$ (see cross-sections in the bottom row of Figs. $3 \mathrm{~m}-3 \mathrm{r}$.).

Third, with increasing field magnitude, particularly for negative fields, larger domains are broken into a large number of small bubble domains (e.g., by comparing Figs. 3o and 3p). Their radius typically shrinks with increasing field magnitude (cross-sections of Figs. 3m-3r) and many domains with a radius below $10 \mathrm{~nm}$ are observed. Note that the measured frequency shift is the convolution of the stray field at a tip height of $10.5 \mathrm{~nm}$ above the surface with the magnetic moment of the MFM tip. Hence the true radius of these bubble domains can be considerably smaller than $10 \mathrm{~nm}$, compatible with the skyrmion size of $\sim 6 \mathrm{~nm}$ expected from the $\mathrm{TH}$ signal. However, if we were to count the number of skyrmions from our MFM images, we would most likely obtain a lower bound as skyrmions smaller than our $10 \mathrm{~nm}$ resolution would not be visible.

The minimal diameter $d_{\min }$ of a magnetostatically-stabilized bubble domain can be calculated $^{30,31}$ as $d_{\min }>\frac{\sigma_{w}}{\mu_{0} H_{c} M}$, where $\sigma_{w}=4 \sqrt{K_{u} \cdot A}$ is the domain wall energy $\left(K_{u}\right.$ : out-ofplane uniaxial anisotropy, $A$ : exchange stiffness), $\mu_{0} H_{c}=2.2 \mathrm{~T}$, and $M$ the SRO magnetization. Using $M \approx 190 \mathrm{kA} / \mathrm{m}$ obtained from our quantitative MFM data analysis (see Fig. 4), $A=2 \mathrm{pJ} / \mathrm{m}$, and $K_{u}=500 \mathrm{~kJ} / \mathrm{m}^{3},{ }^{32}$ the minimum diameter $d_{\min }=9.6 \mathrm{~nm}$. Given the large coercivity of $2.2 \mathrm{~T}$ at $10 \mathrm{~K}, d_{\min }$ can be smaller than $10 \mathrm{~nm}$, even in the absence of DMI. However, at higher 
temperatures where $H_{\mathrm{c}}$ decreases by an order of magnitude, we still detect strong $\mathrm{TH}$ signal and observe nanoscale bubble domains by MFM (see Supplementary Information); thus interfacial DMI must play an important role in the skyrmion formation.
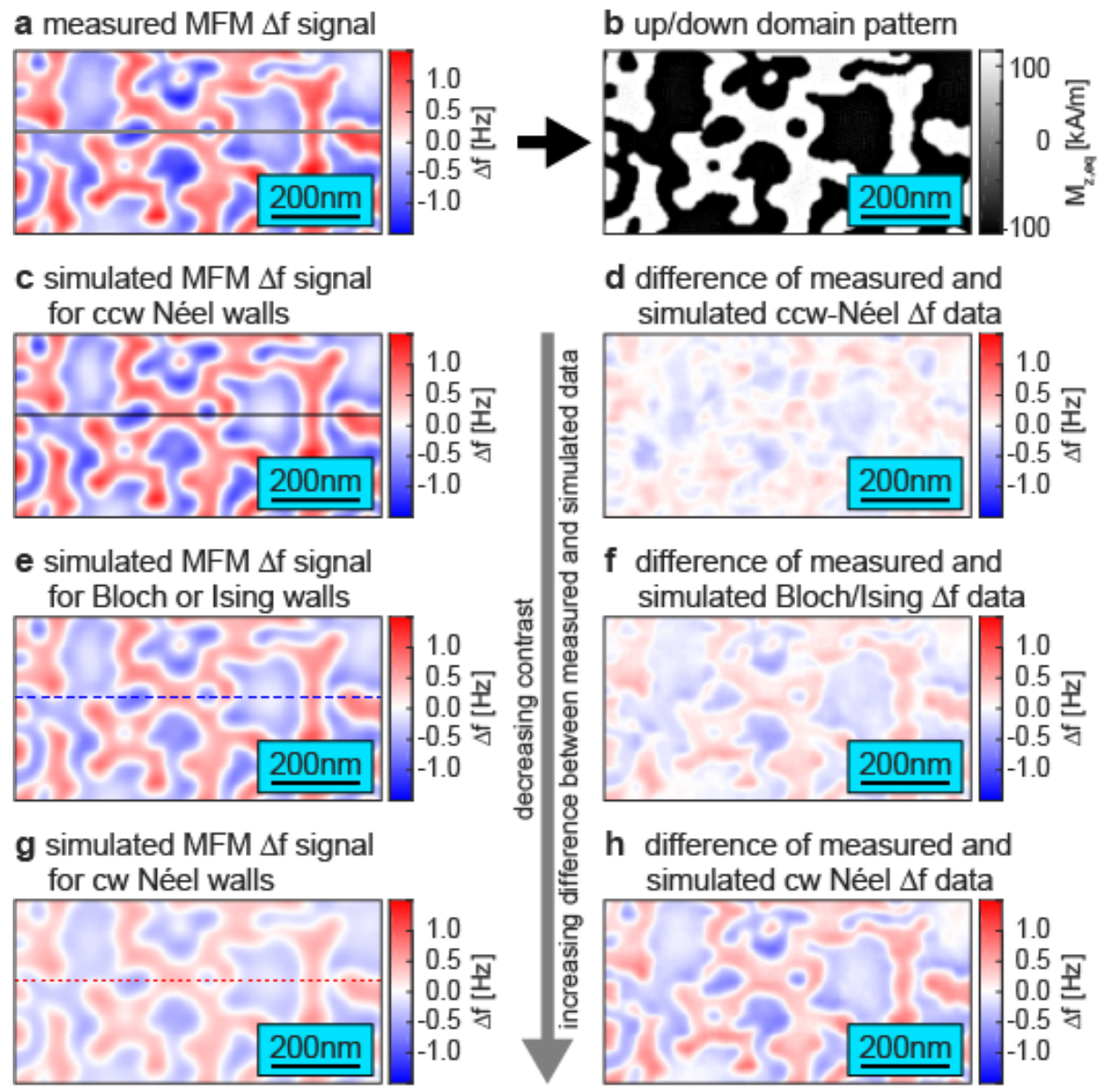

j difference of measured and

i cross sections from a, c, e, g
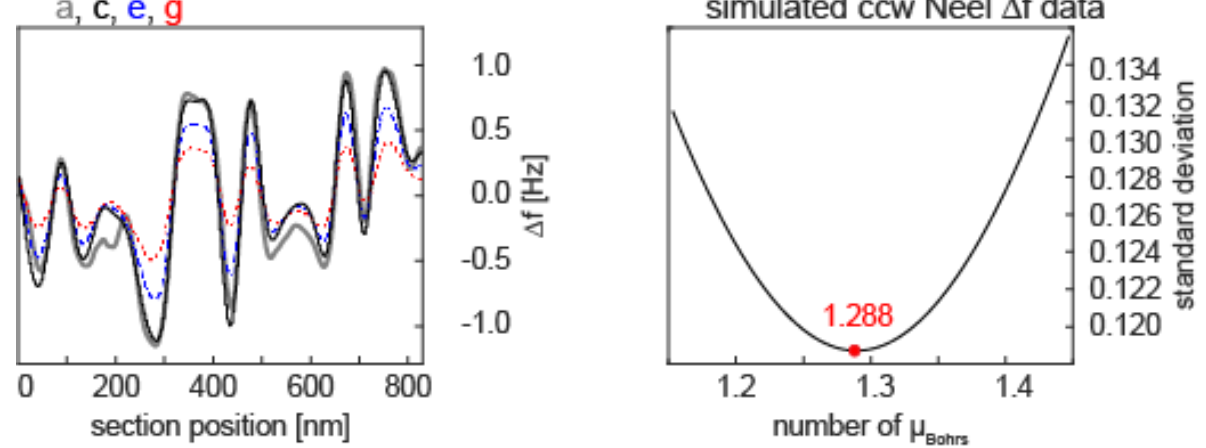

Figure 4. Determination of the sign of the DMI from a quantitative analysis of the MFM images for the $\operatorname{SIO}(2 \mathrm{uc}) / \mathrm{SRO}(10 \mathrm{uc})$ bilayer. a, MFM $\Delta$ f pattern measured at $10 \mathrm{~K}$. b, Pattern of up/down domains obtained by discriminating the data shown in a. c, Modeled MFM $\Delta \mathrm{f}$ pattern 
calculated from the up/down domains in $\mathbf{b}$ using a calibrated MFM tip transfer function, and assuming a magnetic moment density of $1.288 \mu$ в per SRO unit cell with ccw-Néel domain walls. d, Difference between measured MFM pattern in a and modeled MFM pattern in c. The remaining contrast difference is small and the pattern is distinct from the domain pattern in c, confirming a good agreement between the measured and modeled MFM data. e, Modeled MFM $\Delta$ f using 1.288 $\mu$ в per SRO unit cell for Bloch or Ising walls. f, Difference between measured MFM pattern in a and that modeled for Bloch or Ising walls in e. g, Modeled MFM $\Delta$ f for cw-Néel walls using 1.288

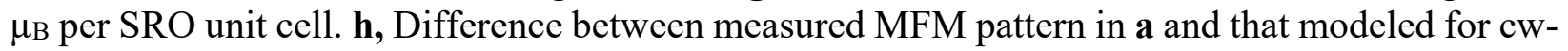
Néel walls in $\mathbf{g}$. Note that the contrast of the modeled MFM data decreases when changing the type of wall from ccw-Néel $\rightarrow$ Bloch/Ising $\rightarrow$ cw-Néel. Conversely, the contrast differences between the measured and simulated patterns increase. i, Cross-sections comparing the measured $\Delta \mathrm{f}$-contrast (thick grey line) to the $\Delta \mathrm{f}$-contrast simulated for the ccw-Néel walls (solid black line), Bloch/Ising walls (dashed blue line), and cw-Néel wall (dotted red line). j, RMS difference of the measured data depicted in $\mathbf{a}$ and the simulated data in $\mathbf{c}$ as a function of the SRO magnetic moment density. The best match is obtained for $1.288 \mu$ B per SRO unit cell.

Because of the strong spin-orbit interaction of the SIO of our SIO/SRO bilayer, we expect the presence of interfacial DMI. Provided that such interfacial DMI is sufficiently strong, the degeneracy of the domain wall orientation is lifted, and the type of wall changes from Bloch to Néel with either counter-clockwise (ccw) or clockwise (cw) orientation, i.e., the bubble domains can become Néel-type skyrmions with a nanoscale core and a helicity that is unity for a ccw-Néel wall and zero for a cw-Néel wall. In contrast to Bloch walls (and also Ising wall which may appear in materials with strong PMA), Néel walls are magnetically charged, i.e., $\rho_{\text {mag }}=-\nabla \cdot \vec{M} \neq 0$ at the locations of the walls. The stray field arising from these magnetic volume charges $\rho_{m a g}$ amplifies (ccw-Néel walls) or attenuates (cw-Néel walls) the field from the magnetic surface charges $\sigma_{\text {mag }}$ arising at the top and bottom surfaces of the SRO layer. ${ }^{10}$ In order to determine the rotation direction of the Néel wall and with it the sign of the interfacial DMI, MFM data obtained at $10 \mathrm{~K}$ of the native domain structure (Fig. 4a) are compared to modeled MFM data for ccw- (Fig. 4c), Bloch or Ising walls (Fig. 4e), and cw-Néel walls (Fig. 4h). The MFM $\Delta$ f signal depends on the interaction of the stray field emanating from the sample surface with the a priori unknown distribution of the tip moments. 
This distribution can be obtained by means of a tip calibration procedure ${ }^{33}$ (see details in Supplementary Information). To calculate the stray field above the sample, a pattern (Fig. 4b) matching the magnetic up/down domains is obtained from the measured MFM $\Delta$ f-pattern (Fig. 4a) by a discrimination procedure. This pattern also describes the pattern of magnetic surface charges at the top and bottom surfaces of the SRO film, $\sigma_{\text {mag }}^{\text {top,bot }}$, and the magnetic volume charges $\rho_{\text {mag }}=$ $-\nabla \cdot \vec{M}$ arising along the Néel but not Bloch or Ising domain walls. From this pattern of magnetic surface and volume charges, and the stray field at a distance of $10.5 \mathrm{~nm}$, the tip-sample separation used in all MFM experiments presented here can be calculated. We use the magnetic moment per SRO unit cell as the sole fitting parameter. Figure $4 \mathrm{c}$ shows a simulated MFM image for an up/down domain pattern (Fig. 4b) with ccw-Néel domain walls using a magnetic moment of 1.288 $\mu_{\text {в }}$ per SRO unit cell obtained by minimizing the root-mean-square (RMS) difference (Fig. $4 \mathrm{j}$ ) between the simulated (Fig. 4c) and the measured (Fig. 4a) $\Delta$ f patterns. For Bloch or Ising walls (Fig. 4e) and also for the cw-Néel walls (Fig. 4g), the field of the surface charge pattern is significantly reduced and a much weaker $\Delta \mathrm{f}$ contrast is obtained. Fig. $4 \mathrm{i}$ compares cross-sections of the measured $\Delta \mathrm{f}$ (thick grey line) to those calculated for ccw-Néel walls (solid black line), Bloch or Ising walls (dashed blue line), and cw-Néel walls (dotted red line), where the $\Delta \mathrm{f}$ contrast for the ccw-Néel wall matches that of the measurement, while that for Bloch or Ising and cw-Néel walls is clearly too weak. In order to obtain a good match of the $\Delta \mathrm{f}$ pattern for Bloch or Ising and cwNéel walls with the measured $\Delta$ f pattern, a magnetic moment of 1.783 and $2.76 \mu$ в per SRO unit cell is required, respectively. Both values are clearly too large compared to that obtained from SQUID measurement. Thus, it is concluded that the domains observed in the zero-field cooled sample must have ccw-Néel walls that arise is the SIO on the top of SRO interface generates a sufficiently strong negative DMI. 
Assuming that the domain wall characteristics does not change with the size of the magnetic object, the observed small bubble domains, which persist over a broad temperature range, can be expected to be "true" skyrmions with a ccw-spin Néel texture that are stabilized both by magnetostatics and the DMI provided by the interface. The small size of the skyrmion is in good agreement with the sizes extracted from the TH signal. This is in stark contrast with the metallic multilayer systems which have several orders of magnitude discrepancies between TH data and real space imaging. ${ }^{34}$ These measurements and analyses indicate that $\mathrm{SrRuO}_{3}$ is a prototypical ferromagnet for studying the correlation between TH effect and skyrmion size.

\section{Methods}

Sample preparation: Epitaxial $\mathrm{SrIrO}_{3} / \mathrm{SrRuO}_{3}$ bilayers were deposited on $\mathrm{SrTiO}_{3}(001)$ by de offaxis sputtering at a substrate temperature of $420^{\circ} \mathrm{C}$ in an $\mathrm{O}_{2} / \mathrm{Ar}$ environment with a total pressure of $8 \mathrm{mTorr}$ ( $7 \mathrm{mTorr}$ ) and an oxygen partial pressure of $96 \mu$ Torr (35 $\mu$ Torr) for SRO (SIO). Prior to growth, STO substrates were treated with buffered-HF for $30 \mathrm{~s}$ and annealed in air at $950^{\circ} \mathrm{C}$ for $1 \mathrm{hr}$ to obtain atomically flat $\mathrm{TiO}_{2}$-terminated surface terraces. SRO and SIO films grown on STO substrates preserve the unit-cell steps, indicating layer-by-layer growth.

Hall measurements: Longitudinal $\left(\rho_{x x}\right)$ and Hall $\left(\rho_{x y}\right)$ resistivity hysteresis loops were measured using a Quantum Design 14 T Physical Property Measurement System (PPMS), with Keithley 2400 SourceMeter and 2182 Nanovoltmeter as current source and voltage detection, respectively. Films were patterned into $0.5 \mathrm{~mm}$-wide, $2 \mathrm{~mm}$-long Hall bars using photolithography and Argon ion milling. A dc current density of $5 \times 10^{8} \mathrm{Am}^{-2}$ with opposite polarities was applied at each data point to average out any thermoelectric effects. Magnetic fields were applied normal to sample surfaces. Hall resistivity loops were anti-symmetrized to exclude longitudinal resistivity contributions from misalignment of the Hall voltage electrodes. 
Magnetization measurements: Magnetic hysteresis loops up to $7 \mathrm{~T}$ were measured using a Quantum Design superconducting quantum interface device (SQUID) magnetometer, with fields applied perpendicular to the sample surface.

MFM experiments: The MFM measurements were performed with a UHV low temperature MFM system that allows the application of fields of up to $7 \mathrm{~T}$ and can be operated in a temperature range from 6 to $120 \mathrm{~K} .{ }^{35}$ The measured quantity is the shift of the resonance frequency of a cantilever that depends on the local force gradients acting on the magnetic tip. To allow a detailed comparison of MFM data taken at different fields, the tip was scanned parallel to the slope of the sample surface with an average tip-sample distance kept constant at $10.5 \mathrm{~nm}$ using frequencymodulated capacitive tip-sample distance control. ${ }^{36}$ Because an MFM measures the sum of all forces acting on the tip, non-magnetic background forces and also magnetic forces stemming from magnetic fields generated by a variation of the magnetic moment areal density are separated (see Supplementary Information) from the measured $\Delta \mathrm{f}$ signal. In this way the data depicted here solely contain contributions arising from the up/down nanoscale domain/skyrmion structures.

Supporting Information: Sample characterizations, Hall measurement analysis and thickness dependence, more details of magnetic force microscopy results and analysis.

Corresponding Author: Fengyuan Yang. Email: yang.1006@osu.edu

Author contributions: K.Y.M. and A.S.A. contributed equally to this work by conducting sample growth, electrical and magnetic measurements, and data analysis. J.F. grew some of the oxide films and did XRD characterization. BDE performed the STEM measurements. M.B., A.O.M., X.Z. and H.J.H performed the MFM measurements and analysis. DWM supervised the STEM characterization. FYY oversaw the whole project. All authors contributed to writing the manuscript. \$These authors contributed equally. 


\section{Acknowledgements}

This work was primarily supported by DARPA under Grant No. D18AP00008. KYM and ASA acknowledge partial support by the Center for Emergent Materials, an NSF-funded MRSEC, under

Grant No. DMR-1420451. FYY acknowledge partial support by the Department of Energy (DOE), Office of Science, Basic Energy Sciences, under Grant No. DE-SC0001304. The MFM work was supported from the Swiss National Science Foundation Sinergia Proposal CRSII2_15441 and DACH proposal 200021E-160637.

\section{REFERENCES}

1. Heinze, S., von Bergmann, K., Menzel, M., Brede, J., Kubetzka, A., Wiesendanger, R., Bihlmayer, G. and Blugel, S., "Spontaneous atomic-scale magnetic skyrmion lattice in two dimensions," Nat. Phys. 2011, 7, 713-718.

2. Boulle, O., Vogel, J., Yang, H. X., Pizzini, S., de Souza Chaves, D., Locatelli, A., Menteş, T. O., Sala, A., Buda-Prejbeanu, L. D., Klein, O., Belmeguenai, M., Roussigné, Y., Stashkevich, A., Chérif, S. M., Aballe, L., Foerster, M., Chshiev, M., Auffret, S., Miron, I. M. and Gaudin, G., "Room-temperature chiral magnetic skyrmions in ultrathin magnetic nanostructures," Nat. Nanotechnol. 2016, 11, 449-454.

3. Jiang, W. J., Upadhyaya, P., Zhang, W., Yu, G. Q., Jungfleisch, M. B., Fradin, F. Y., Pearson, J. E., Tserkovnyak, Y., Wang, K. L., Heinonen, O., te Velthuis, S. G. E. and Hoffmann, A., "Blowing magnetic skyrmion bubbles," Science 2015, 349, 283-286.

4. Moreau-Luchaire, C., Moutafis, C., Reyren, N., Sampaio, J., Vaz, C. A. F., Van Horne, N., Bouzehouane, K., Garcia, K., Deranlot, C., Warnicke, P., Wohlhuter, P., George, J. M., Weigand, M., Raabe, J., Cros, V. and Fert, A., "Additive interfacial chiral interaction in multilayers for stabilization of small individual skyrmions at room temperature," Nat. Nanotechnol. 2016, 11, 444-448.

5. Woo, S. H., Litzius, K., Krüger, B., Im, M.-Y., Caretta, L., Richter, K., Mann, M., Krone, A., Reeve, R. M., Weigand, M., Agrawal, P., Lemesh, I., Mawass, M.-A., Fischer, P., Kläui, M. and Beach, G. S. D., "Observation of room-temperature magnetic skyrmions and their currentdriven dynamics in ultrathin metallic ferromagnets," Nat. Mater. 2016, 15, 501-506.

6. Yu, X. Z., Kanazawa, N., Zhang, W. Z., Nagai, T., Hara, T., Kimoto, K., Matsui, Y., Onose, Y. and Tokura, Y., "Skyrmion flow near room temperature in an ultralow current density," Nat. Commun. 2012, 3, 988.

7. Kanazawa, N., Seki, S. and Tokura, Y., "Noncentrosymmetric Magnets Hosting Magnetic Skyrmions," Adv. Mater. 2017, 29, 1603227.

8. Fert, A., Reyren, N. and Cros, V., "Magnetic skyrmions: advances in physics and potential applications," Nat. Rev. Mater. 2017, 2, 17031. 
9. Wiesendanger, R., "Nanoscale magnetic skyrmions in metallic films and multilayers: a new twist for spintronics," Nat. Rev. Mater. 2016, 1, 16044.

10. Marioni, M. A., Penedo, M., Baćani, M., Schwenk, J. and Hug, H. J., "Halbach Effect at the Nanoscale from Chiral Spin Textures," Nano Lett. 2018, 18, 2263-2267.

11. Matsuno, J., Ogawa, N., Yasuda, K., Kagawa, F., Koshibae, W., Nagaosa, N., Tokura, Y. and Kawasaki, M., "Interface-driven topological Hall effect in $\mathrm{SrRuO}_{3}-\mathrm{SrIrO}_{3}$ bilayer," Sci. Adv. 2016, 2, e1600304.

12. Ohuchi, Y., Matsuno, J., Ogawa, N., Kozuka, Y., Uchida, M., Tokura, Y. and Kawasaki, M., "Electric-field control of anomalous and topological Hall effects in oxide bilayer thin films," Nat. Commun. 2018, 9, 213.

13. Vistoli, L., Wang, W. B., Sander, A., Zhu, Q. X., Casals, B., Cichelero, R., Barthélémy, A., Fusil, S., Herranz, G., Valencia, S., Abrudan, R., Weschke, E., Nakazawa, K., Kohno, H., Santamaria, J., Wu, W. D., Garcia, V. and Bibes, M., "Giant topological Hall effect in correlated oxide thin films," Nat. Phys. 2019, 15, 67-72.

14. Wang, L. F., Feng, Q. Y., Kim, Y. K., Kim, R., Lee, K. H., Pollard, S. D., Shin, Y. J., Zhou, H. B., Peng, W., Lee, D., Meng, W. J., Yang, H., Han, J. H., Kim, M., Lu, Q. Y. and Noh, T. W., "Ferroelectrically tunable magnetic skyrmions in ultrathin oxide heterostructures," Nat. Mater. 2018, 17, 1087-1094.

15. Kan, D., Moriyama, T., Kobayashi, K. and Shimakawa, Y., "Alternative to the topological interpretation of the transverse resistivity anomalies in $\mathrm{SrRuO}_{3}$," Phys. Rev. B 2018, 98, 180408.

16. Morikawa, D., Yu, X. Z., Kaneko, Y., Tokunaga, Y., Nagai, T., Kimoto, K., Arima, T. and Tokura, Y., "Lorentz transmission electron microscopy on nanometric magnetic bubbles and skyrmions in bilayered manganites $\mathrm{La}_{1.2} \mathrm{Sr}_{1.8}\left(\mathrm{Mn}_{1-\mathrm{y}} \mathrm{Ru}_{\mathrm{y}}\right)_{2} \mathrm{O}_{7}$ with controlled magnetic anisotropy," Appl. Phys. Lett. 2015, 107, 212401.

17. Onose, Y., Okamura, Y., Seki, S., Ishiwata, S. and Tokura, Y., "Observation of Magnetic Excitations of Skyrmion Crystal in a Helimagnetic Insulator $\mathrm{Cu}_{2} \mathrm{OSeO}_{3}$," Phys. Rev. Lett. 2012, 109, 037603.

18. Hauser, A. J., Williams, R. E. A., Ricciardo, R. A., Genc, A., Dixit, M., Lucy, J. M., Woodward, P. M., Fraser, H. L. and Yang, F. Y., "Unlocking the potential of half-metallic $\mathrm{Sr}_{2} \mathrm{FeMoO}_{6}$ films through controlled stoichiometry and double-perovskite ordering," Phys. Rev. B 2011, 83, 014407.

19. Du, C. H., Wang, H. L., Hammel, P. C. and Yang, F. Y., " $\mathrm{Y}_{3} \mathrm{Fe}_{5} \mathrm{O}_{12}$ Spin Pumping for Quantitative Understanding of Pure Spin Transport and Spin Hall Effect in a Broad Range of Materials (Invited)," J. Appl. Phys. 2015, 117, 172603.

20. Yang, F. Y. and Hammel, P. C., "Topical review: FMR-Driven Spin Pumping in $\mathrm{Y}_{3} \mathrm{Fe}_{5} \mathrm{O}_{12}-$ Based Structures," J. Phys. D: Appl. Phys. 2018, 51, 253001.

21. Neubauer, A., Pfleiderer, C., Binz, B., Rosch, A., Ritz, R., Niklowitz, P. G. and Boni, P., "Topological Hall Effect in the A Phase of MnSi," Phys. Rev. Lett. 2009, 102, 186602.

22. Gallagher, J. C., Meng, K. Y., Brangham, J. T., Wang, H. L., Esser, B. D., McComb, D. W. and Yang, F. Y., "Robust Zero-Field Skyrmion Formation in FeGe Epitaxial Thin Films," Phys. Rev. Lett. 2017, 118, 027201.

23. Soumyanarayanan, A., Raju, M., Gonzalez Oyarce, A. L., Tan, A. K. C., Im, M.-Y., Petrović, A. P., Ho, P., Khoo, K. H., Tran, M., Gan, C. K., Ernult, F. and Panagopoulos, C., "Tunable room-temperature magnetic skyrmions in $\mathrm{Ir} / \mathrm{Fe} / \mathrm{Co} / \mathrm{Pt}$ multilayers," Nat. Mater. 2017, 16, 898904. 
24. Ahmed, A. S., Rowland, J., Esser, B. D., Dunsiger, S. R., McComb, D. W., Randeria, M. and Kawakami, R. K., "Chiral bobbers and skyrmions in epitaxial FeGe/Si(111) films," Phys. Rev. Mater. 2018, 2, 041401.

25. Bruno, P., Dugaev, V. K. and Taillefumier, M., "Topological Hall Effect and Berry Phase in Magnetic Nanostructures," Phys. Rev. Lett. 2004, 93, 096806.

26. Huang, S. X. and Chien, C. L., "Extended Skyrmion Phase in Epitaxial FeGe(111) Thin Films," Phys. Rev. Lett. 2012, 108, 267201.

27. Worledge, D. C. and Geballe, T. H., "Negative Spin-Polarization of SrRuO3," Phys. Rev. Lett. 2000, 85, 5182-5185.

28. Porthun, S., Abelmann, L. and Lodder, C., "Magnetic force microscopy of thin film media for high density magnetic recording," J. Magn. Magn. Mater. 1998, 182, 238-273.

29. Baćani, M., Marioni, M. A., Schwenk, J. and Hug, H. J., "How to measure the local Dzyaloshinskii-Moriya Interaction in Skyrmion Thin-Film Multilayers," Sci. Rep. 2019, 9, 3114.

30. Huth, B. G., "Calculations of stable domain radii produced by thermomagnetic writing," IBM J. Res. Dev. 1974, 18, 100-109.

31. Tsunashima, S., "Magneto-optical recording," J. Phys. D: Appl. Phys. 2001, 34, R87-R102.

32. Ziese, M., Vrejoiu, I. and Hesse, D., "Inverted hysteresis and giant exchange bias in La0.7 $\mathrm{Sr}_{0.3} \mathrm{MnO}_{3} / \mathrm{SrRuO}_{3}$ superlattices," Appl. Phys. Lett. 2010, 97, 052504.

33. van Schendel, P. J. A., Hug, H. J., Stiefel, B., Martin, S. and Güntherodt, H. J., "A method for the calibration of magnetic force microscopy tips," J. Appl. Phys. 2000, 88, 435-445.

34. Maccariello, D., Legrand, W., Reyren, N., Garcia, K., Bouzehouane, K., Collin, S., Cros, V. and Fert, A., "Electrical detection of single magnetic skyrmions in metallic multilayers at room temperature," Nat. Nanotechnol. 2018, 13, 233-237.

35. Hug, H. J., Stiefel, B., van Schendel, P. J. A., Moser, A., Martin, S. and Güntherodt, H. J., "A low temperature ultrahigh vaccum scanning force microscope," Rev. Sci. Instrum. 1999, 70, 3625-3640.

36. Zhao, X., Schwenk, J., Mandru, A. O., Penedo, M., Baćani, M., Marioni, M. A. and Hug, H. J., "Magnetic force microscopy with frequency-modulated capacitive tip-sample distance control," New J. Phys. 2018, 20, 013018. 